\title{
Clinical and virologic outcomes after changes in first antiretroviral regimen at 7 sites in the Caribbean, Central and South America Network (CCASAnet)
}

\author{
Marcelo Wolff, M.D. ${ }^{1}$, Bryan E. Shepherd, Ph.D. ${ }^{2}$, Claudia Cortés, M.D. ${ }^{1}$, Peter Rebeiro, \\ M.H.S., Ph.D. ${ }^{2}$, Carina Cesar, M.D. ${ }^{3}$, Sandra Wagner Cardoso, M.D., Ph.D. ${ }^{4}$, Jean W. Pape, \\ M.D. ${ }^{5}$, Denis Padgett, M.D. ${ }^{6}$, Juan Sierra-Madero, M.D. ${ }^{7}$, Juan Echevarria, M.D. ${ }^{8}$, and \\ Catherine C. McGowan, M.D. ${ }^{2}$ for The Caribbean, Central and South America Network for \\ HIV Epidemiology (CCASAnet) \\ ${ }^{1}$ Fundación Arriarán/Facultad de Medicina, Universidad de Chile, Santiago, Chile \\ ${ }^{2}$ Vanderbilt University, Nashville, TN, U.S.A. \\ ${ }^{3}$ Fundación Huésped, Buenos Aires, Argentina \\ ${ }^{4}$ Instituto de Pesquisa Clinica Evandro Chagas-Fundação Oswaldo Cruz, Rio de Janeiro, Brazil \\ ${ }^{5}$ Le Groupe Haïtien d'Etude du Sarcome de Kaposi et des Infections Opportunistes, Port-au- \\ Prince, Haiti \\ ${ }^{6}$ Instituto Hondureño de Seguridad Social and Hospital Escuela, Tegucigalpa, Honduras \\ ${ }^{7}$ Instituto Nacional de Ciencias Médicas y Nutrición, Mexico City, México \\ 8Instituto de Medicina Tropical Alexander von Humboldt, Lima, Peru
}

\section{Abstract}

Background-HIV-infected persons in lower income countries may experience high rates of antiretroviral therapy (ART) change, particularly due to toxicity or other non-failure reasons. Few reports address patient outcomes after these modifications.

\begin{abstract}
Methods-HIV-infected adults from 7 Caribbean, Central and South America network (CCASAnet) clinical cohorts who modified $>$ or $=1$ drug from first ART regimen (ART-1) for any reason thereby starting a second regimen (ART-2) were included.
\end{abstract}

\begin{abstract}
Results-5,565 ART-naïve HAART initiators started ART-2 after a median of 9.8 months on ART-1; 39\% changed to ART-2 due to toxicity and $11 \%$ due to failure. Median follow-up after starting ART-2 was 2.9 years; $45 \%$ subsequently modified ART-2. Cumulative incidences of death at 1,3 , and 5 years after starting ART-2 were $5.1 \%, 8.4 \%$ and $10.5 \%$, respectively. In adjusted analyses, death was associated with older age, clinical AIDS, lower CD4 at ART-2 start, earlier calendar year, and starting ART-2 because of toxicity (adjusted hazard ratio[aHR]=1.5 vs.
\end{abstract}

Corresponding author: Catherine C. McGowan, M.D., A2200 Medical Center North, Division of Infectious Diseases, Vanderbilt University Medical Center, Nashville, TN 37232, Tel: (615) 322-2035, Fax: (615) 343-6160, c.mcgowan@ vanderbilt.edu.

Some of the data herein were presented as an oral abstract at the HIV Drug Therapy in the Americas conference, held June 13-15, 2013 in Sao Paulo, Brazil. 
failure, $95 \%$ confidence interval[CI $]=1.0-2.1)$. Cumulative incidences of $\mathrm{VF}$ after 1, 3, and 5 years were $9 \%, 19 \%$, and $25 \%$. In adjusted analyses, $\mathrm{VF}$ was associated with younger age, earlier calendar year, lower CD4 at start of ART-2, and starting ART-2 because of failure (aHR=2.1 vs. toxicity, $95 \% \mathrm{CI}=1.5-2.8)$.

Conclusions-Among patients modifying first ART regimen, risks of subsequent modifications, mortality, and virologic failure were high. Access to improved antiretrovirals in the region is needed to improve initial treatment success.

\section{Keywords}

antiretroviral agents; treatment failure; cohort studies; Latin America; Caribbean region

\section{Introduction}

Highly active antiretroviral therapy (HAART) has significantly reduced morbidity and mortality in persons living with HIV worldwide (1-5), and become more widely available in resource-limited settings. An estimated 12.9 million people globally, and 11.7 million from low- and middle-income countries, were receiving HAART by 2014 (6).

The HIV epidemic in Latin America and the Caribbean accounts for approximately 6\% of HIV-infected persons worldwide, an estimated $70 \%$ of whom are aware of diagnosis and $44 \%$ of whom are receiving HAART in the region (6-8). Expanded access programs to HAART have been in place in Latin America for over a decade, leading to a regional coverage of $56 \%$ of those eligible by the end of $2013(8,9)$.

Despite the successes of HAART, high incidence of early mortality (10-13) and frequent change of initial therapy $(14,15)$ have been consistently observed in many regions, including Latin America and the Caribbean. HAART initiation at more advanced disease stages may lead to poorer tolerability, slower and blunted treatment response, and higher risk of disease progression and mortality $(11,16)$. HAART-related toxicities and intolerance may lead to poor adherence, viral resistance, virologic failure, and diminished likelihood of future treatment success. Many studies have shown the frequent need for partial or total change of initial regimens, with toxicity, more frequently than failure, being the main reason $(8,14,17-$ 19). However, clinical and virologic outcomes while on those modified regimens, except when changed due to failure, are not well-characterized in "real world" settings. Most current understanding comes from studies of specific drug combinations or controlled scenarios, usually after failure of initial therapy (20-27). Few studies, though, have investigated outcomes after initial regimen modification due to toxicity or other non-failure reasons, the largest being from cohorts in high-income countries (28). Evaluation of outcomes after changes in first HAART in routine clinical practice is urgently needed and was the main objective of this study. We evaluated the cumulative incidence of regimen modification, mortality, and virologic failure while on a second HAART regimen among patients in Latin America and the Caribbean who changed their first HAART regimen for any reason. The association between the reason for change and effectiveness and durability of subsequent regimens also was examined. 


\section{Methods}

The Caribbean, Central and South America network for HIV epidemiology (CCASAnet; www.ccasanet.org) is a consortium of adult HIV clinics from 7 countries established as part of the International Epidemiologic Databases to Evaluate AIDS (IeDEA; www.iedea.org) (29). CCASAnet sites contributing data to this study were Hospital Fernandez and Centro Médico Huésped, Buenos Aires, Argentina (HF/CMH-Argentina); Instituto de Pesquisa Clinica Evandro Chagas, Fundação Oswaldo Cruz, Rio de Janeiro, Brazil (FC- Brazil); Fundación Arriarán, Santiago, Chile (FA-Chile); Le Groupe Haïtien d'Etude du Sarcome de Kaposi et des Infections Opportunistes, Port-au-Prince, Haiti (GHESKIO-Haiti); Instituto Hondureño de Seguridad Social and Hospital Escuela, Tegucigalpa, Honduras (IHSS/HEHonduras); Instituto Nacional de Ciencias Médicas y Nutrición Salvador Zubirán, Mexico City, Mexico (INCMNSZ-Mexico); and Instituto de Medicina Tropical Alexander von Humboldt, Lima, Peru (IMTAvH-Peru). Clinical and epidemiological data were collected at each site, de-identified, and sent to the CCASAnet Data Coordinating Center at Vanderbilt University (VDCC; Nashville, Tennessee, USA), for data harmonization. Data quality checks and on-site audits were performed by VDCC to ensure data accuracy. Institutional review board approval was obtained from each site and from Vanderbilt University.

Antiretroviral-naïve adults ( $\geq 18$ years) initiating their first HAART regimen (ART-1) between 1996 and 2014 at participating sites and subsequently modifying at least one drug, thereby starting a second regimen (ART-2), were eligible for inclusion. Patients participating in any clinical intervention protocol were excluded from analyses. All patients from IMTAvH-Peru were included in analyses though clinical trial participation was not documented, but estimated as less than $5 \%$.

Clinical AIDS at initiation of ART-1 was defined as CDC stage C, WHO stage IV, or a specification of AIDS. HAART was defined as protease inhibitor (PI)-based (one ritonavirboosted or unboosted PI plus two nucleoside reverse transcriptase inhibitors [NRTI]), nonnucleoside reverse transcriptase inhibitor (NNRTI)-based (one NNRTI plus two NRTIs), or other combinations (including triple NRTI regimens and all other regimens containing at least three drugs), in accordance with national or international guidelines over the study period.

Nadir pre-HAART CD4 ${ }^{+}$lymphocyte count (CD4) was the lowest measurement prior to, or no more than seven days after, initiating ART-1. CD4 at start of ART-2 was the measurement closest to, but no more than 180 days before, or seven days after. Pre-HAART HIV-1 RNA viral load (VL) was defined as the measurement closest to ART-1 initiation, but no more than 180 days before; VL measurements after ART-1 initiation were not included. VL at start of ART-2 was defined using the same time intervals.

Study outcomes included all-cause mortality, modification of ART-2 regimen, and virologic failure. Virologic failure was defined as one of: 1) VL was never $<400$ copies $/ \mathrm{mL}$ after six months of therapy; 2) VL was once $<400$ copies $/ \mathrm{mL}$, but two consecutive measurements subsequently were $>400$ copies $/ \mathrm{mL}$; 3) VL was once $<400$ copies $/ \mathrm{mL}$, but a single measurement subsequently was $>1000$ copies $/ \mathrm{mL}$. The cutoff of 400 copies $/ \mathrm{mL}$ was the 
lower limit of quantitation for assays in use at many of the sites during the study period. Patients modifying ART-2 before virologic failure were censored at the time of regimen modification for virologic failure analyses. Loss to follow-up (LTFU) was defined as no record of visit (clinic, laboratory, or pharmacy) within the year prior to the site-specific database closing date; patients who were lost to follow-up were censored at last visit.

The probability of death after starting ART-2 was estimated using Kaplan-Meier methods, with time 0 defined as the start of ART-2. When estimating the cumulative incidences of LTFU, regimen change, and virologic failure as events of interest, death was treated as a competing event $(30,31)$. Risk factors for death, virologic failure, and change of ART-2 were assessed using Cox proportional hazards models. All models were stratified by CCASAnet site. Primary adjusted models included sex, age at start of ART-2, CD4 at start of ART-2, change in CD4 from ART-1 to ART-2, year of ART-2 start, months between start of ART-1 and start of ART-2, clinical AIDS at start of ART-1, and primary reason for changing regimens (classified as toxicity, failure, other, or unknown). These covariates were chosen for inclusion a priori based on perceived clinical relevance and availability. Adjusted analyses used multiple imputation to account for missing CD4 and clinical AIDS values (32); 10 imputation replications were used. Age and CD4 were included in the models using restricted cubic splines with 4 knots to relax linearity assumptions (33); CD4 was also square root-transformed. The time between the start of ART-1 and ART-2 was logtransformed and included using splines with 3 knots. Associations between continuous variables and outcomes were assessed using p-values from likelihood ratio tests; hazard ratio estimates and 95\% confidence intervals (95\% CI) are given in the text and tables for selected covariate levels. Proportional hazards were assessed using Schoenfeld residuals and a correlation-with-time test (34). There was some evidence that hazards between reasons for changing to ART-2 were not proportional in the mortality analysis; results were similar when separate baseline hazards were assumed for reasons for changing to ART-2.

GHESKIO-Haiti did not routinely measure VL and was excluded from analyses of virologic failure. All analyses were performed using R Statistical Software; analysis scripts are available at http://biostat.mc.vanderbilt.edu/ArchivedAnalyses.

\section{Results}

Of 15,006 ART-naive HAART initiators, 5565 (37\%) met inclusion criteria and started ART-2. Patient characteristics, stratified by reason for starting ART-2, are in Table 1. Fiftyeight percent of patients were male and median age at start of ART-2 was 38 years (interquartile range [IQR] 32-46). CD4 at start of ART-2 was low (median 196 cells/ $\mu \mathrm{L}$; IQR 82-336), but was higher than nadir CD4 prior to starting ART-1 (median 134 cells $/ \mu \mathrm{L}$; IQR 52-226). Median CD4 change from ART-1 to ART-2 was +60 cells/ $\mu \mathrm{L}$ (IQR 0-209). Approximately $30 \%$ of patients had AIDS at start of ART-1; clinical stage at start of ART-2 was not reliably collected across sites and was not included.

Thirty-nine percent of patients started ART-2 due to toxicity of previous regimen; hematologic toxicities were most common, comprising $32 \%$ of toxicity-motivated switches. Eleven percent started ART-2 due to failure of ART-1: $4.5 \%$ due to virologic failure and $6.7 \%$ due to clinical/immunologic failure. Forty-four percent started ART-2 for other 
reasons (availability of preferred agent/regimen, 20\%; drug interactions/co-morbidities, 5\%; unavailability of drugs, $3 \%$; pregnancy-related, $3 \%$; abandonment/non-adherence, $1 \%$ ).

Reason for changing to ART-2 was unknown for $6 \%$ of patients. More details on reasons for changing from ART-1 to ART-2 are provided in the Supplementary Material. Median time on ART-1 prior to ART-2 was 9.8 months (IQR 2.9-31.5 months); this varied substantially by reasons for starting ART-2 (medians of 3.6, 31.4, 12.1, and 13.1 months for those who changed due to toxicity, failure, other, and unknown reasons, respectively). The majority (68\%) of second regimens were NNRTI-based (44\% efavirenz, 25\% nevirapine), although boosted PIs (particularly those containing lopinavir) accounted for $80 \%$ of second regimens among those who started ART-2 due to failure. Most common NRTI drugs in ART-2 were lamivudine (88\%), zidovudine (38\%), tenofovir (34\%), stavudine (15\%), abacavir (14\%), didanosine $(7 \%)$, and emtricitabine $(7 \%)$. These data are shown in the Supplementary Material.

Median follow-up after starting ART-2 was 2.9 years (IQR 1.3-5.8). Among patients starting ART-2, 14.8\% were lost to follow up; cumulative incidence of LTFU three years after starting ART-2 was $11.6 \%$. Comparison of LTFU vs. active patients at all sites is included in the Supplemental Material.

A total of 2,474 patients (45\%) stopped ART-2 during follow-up; 2,340 of these patients (95\%) started a third regimen during follow-up. Cumulative incidences of stopping ART-2 were $24 \%, 44 \%$, and $57 \%$ one, three, and five years after starting ART-2. Cumulative incidences of stopping ART-2 three years after starting ART-2 were fairly similar across reasons for starting ART-2: 48\%, 43\%, 41\%, and 44\% for toxicity, failure, other, and unknown, respectively. Common reasons for stopping ART-2 included toxicity (29\%), failure (11\%), availability of a preferred agent/regimen (19\%), unavailability of drugs (6\%), drug interactions/co-morbidities (5\%), pregnancy-related (4\%), and abandonment/nonadherence (4\%); details are given in the Supplementary Material. The majority (54\%) of third regimens were NNRTI-based (36\% efavirenz, 19\% nevirapine), 36\% were boosted PIs, and the most common NRTI drugs were lamivudine (81\%), tenofovir (37\%), zidovudine (35\%), stavudine (11\%), abacavir (21\%), emtricitabine (9\%), and didanosine (9\%).

A total of 494 (8.9\%) patients died after starting ART-2. Estimated probabilities of mortality one, three, and five years after starting ART-2 were 0.051, 0.084, and 0.105, respectively. Figure 1 shows estimated probabilities of mortality, by study site and reason for starting ART-2. Among those with a known reason, those who changed due to toxicity had the highest risk of mortality during the first several years of ART-2, though mortality risk was similar between those switching due to toxicity and failure by 5 years $(0.117$ and 0.116 , respectively). Mortality was consistently lower for those changing due to "other" reasons.

Risk factors for mortality after starting ART-2 are shown in Table 2. Compared with patients who changed due to toxicity, those who changed due to failure had a lower hazard of death (adjusted HR [aHR] $=0.69,95 \%$ CI $0.47-1.00$ ). Patients who changed for unknown reasons had a higher hazard (aHR=1.56, 95\% CI 1.11-2.20). Patients changing ART-1 due to hematological toxicity had a higher hazard of mortality than those changing due to other toxicities (aHR=1.43; 95\% CI 1.08-1.88; not shown in Table 2). Compared to a patient 
starting ART-2 with CD4 $=350$ cells $/ \mu \mathrm{L}$, the estimated hazards of death for a patient starting ART-2 with CD4=50, 100, or 200 cells/ $\mu \mathrm{L}$ were 3.0 (95\% CI 2.1-4.4), 2.4 (95\% CI 1.73.4), and 1.5 (95\% CI 1.2-1.8) times greater, respectively, holding all other variables equal. Change in CD4 from ART-1 to ART-2 was also a strong predictor of mortality ( $\mathrm{p}=0.006)$, with patients whose CD4 decreased or only slightly increased being at higher risk of death than those whose CD4 substantially increased. AIDS prior to ART-1 and older age were also associated with mortality.

Cumulative incidences of virologic failure while on ART-2, by study site and by reason for starting ART-2, are shown in Figure 2. Cumulative incidences of virologic failure one, three, and five years after starting ART-2 were $9.1 \%, 18.9 \%$, and $24.8 \%$, respectively, although risks were variable by site, ranging at 5 years from a low of 19\% in IHSS/HE-Honduras and INCMNSZ-Mexico to a high of 35\% in FC-Brazil. Median frequency of VL measurements ranged from 1.0 per year (IHSS/HE-Honduras) to 2.4 per year (INCMNSZ-Mexico). The cumulative incidence of virologic failure five years after changing ART-1 due to failure was high: $45 \%$ compared to $20 \%, 26 \%$, and $22 \%$ for those with toxicity, other, or unknown reasons for change.

In analyses controlling for patient characteristics (Table 3), reason for starting ART-2 was strongly associated with virologic failure while on ART-2 ( $\mathrm{p}<0.001)$. The hazard of virologic failure on ART-2 for a patient starting ART-2 due to failure of ART-1 was 2.06 times that of a patient starting ART-2 due to toxicity (95\% CI 1.52-2.79). Younger age, earlier calendar year, lower CD4 at ART-2 initiation, and smaller change in CD4 from ART-1 to ART-2 were also independently associated with an increased hazard of virologic failure.

Associations between specific antiretroviral drugs used in ART-2 and regimen modification, mortality, and virologic failure are provided in the Supplemental Material. Compared to patients with ART-2 containing zidovudine, patients with ART-2 containing tenofovir had lower hazards of mortality (aHR $=0.48,95 \%$ CI $0.33-0.71$ ) and further regimen modification (aHR $=0.78,95 \%$ CI $0.67-0.90$ ). In contrast, patients starting a second regimen containing stavudine or didanosine had higher hazards of mortality (aHR=1.84, 95\% CI 1.43-2.36; $1.64,95 \%$ CI 1.11-2.44; respectively) and subsequent regimen change (aHR=2.06, 95\% CI $1.82-2.32 ; 1.28,95 \%$ CI 1.03-1.58; respectively). Compared to patients with ART-2 containing zidovudine, those with ART-2 containing abacavir had a higher hazard of death (aHR=2.05, 95\% CI 1.39-3.04) and lower hazard of virologic failure (aHR $=0.61,95 \% \mathrm{CI}$ $0.41-0.90)$ or regimen change $(\mathrm{aHR}=0.81,95 \%$ CI $0.68-0.97)$. Those with ART-2 containing efavirenz had a slightly lower hazard of virologic failure $(\mathrm{aHR}=0.75,95 \% \mathrm{CI}$ 0.57-1.00) than those with ART-2 containing nevirapine. Patients with ART-2 containing ritonavir-boosted indinavir or saquinavir had higher hazards of subsequent change (aHR=2.23, 95\% CI 1.50-3.31; aHR=1.50, 95\% CI 1.08-2.09) than those with ART-2 containing ritonavir-boosted lopinavir. 


\section{Discussion}

This study evaluated multiple outcomes after modifying at least one drug in an initial ART regimen for any reason in patients from a large Latin American and Caribbean cohort, and compared outcomes according to the reason for change from ART-1 to ART-2. Significant risk of second regimen change, virologic failure, and mortality were observed after modifications of initial ART regimen. Reasons for changing the second regimen were often similar to those for changing the first; changing to a second regimen due to failure was a strong predictor of subsequent virologic failure. Persons changing due to toxicity had a higher risk of death than persons changing due to failure.

There are many studies of outcomes after first HAART change due to treatment failure $(18,20-27,35-37)$, primarily involving change in most or all of the previous drugs in use. However, there are few studies of outcomes following first HAART change of any drug in the initial regimen due to non-failure reasons (28).

Nearly $40 \%$ of patients in our study changed their initial regimen due to toxicity/intolerance. Toxicity has been consistently reported as the main reason for change of first HAART globally $(14,17,19,38,39)$, although many of these earlier studies involved treatment with older, more toxic antiretroviral agents such as didanosine, stavudine, indinavir or nevirapine. While these drugs are now mostly outdated in developed countries, they were frequently included in initial regimens in our study, as the NRTI components of HAART have not changed considerably in resource-limited settings, including Latin America and the Caribbean.

ART regimen change due to toxicity usually implicates a specific drug and, in the absence of other reasons for poor adherence, such changes should not jeopardize future response to therapy. This of course assumes that clinicians have multiple less toxic alternatives available under these circumstances. Interestingly, changing due to toxicity was associated with increased early mortality on ART-2 in our study. Patients who changed to ART-2 due to a hematologic toxicity had a particularly high risk of death. This suggests the need for broader access to newer antiretrovirals that have improved tolerability (19). In addition, the predictive power of patient outcomes on ART-1 for the most serious adverse events, even after changes in therapy, highlights the potential benefit of focusing resources to enhance monitoring of those patients experiencing blunted CD4 response, and of course, toxicities while on their initial regimen. Though this may seem an obvious course of action and is essentially in line with recent recommendations for first line therapy by the United States (40) and the World Health Organization (41), the execution and logistics involved are not trivial in resource-limited settings (42). Further, despite the implication of these findings that prescription of an initial regimen with maximal antiviral activity and a low side-effect/ toxicity profile would be the surest bulwark against accelerated disease progression, again, the plausibility of implementing this practice is greatly dependent on the most readily available antiretroviral agents $(43,44)$. In some of the countries with contributing cohorts for this study, the ability to initiate ART-1 using second-generation NNRTIs, integrase inhibitors, fixed-dose combination ART excluding older NRTI backbones, or other welltolerated yet potent regimens, is not yet fully realized; the cost of newer regimens is a major 
barrier to their use. For example, in 2012-2014, over $25 \%$ of patients in this study started an initial ART regimen including zidovudine (data not shown).

Mortality in the first year after starting ART-2 was $5.1 \%$, less than that observed in the first year after starting ART-1, estimated as $8 \%$ in an earlier CCASAnet study (11), but still fairly high. Early mortality after starting ART-2 and throughout follow-up was quite variable across sites, illustrating the remarkable heterogeneity across the region. Loss to follow-up of patients on ART-2 was high (15\% overall), and our results suggest that better approaches for patient retention and linkage are needed.

Not surprisingly, known predictors of mortality at HAART initiation (including lower CD4, older age, and AIDS) remained predictors of mortality among patients starting a second regimen. Patients with an unknown reason for starting ART-2 were observed to have the highest risk of mortality, possibly reflecting data collection issues or less engagement in care. Patients who modified their regimens for a known reason other than failure or toxicity had the lowest risk of mortality, which is not to be unexpected given that nearly half of these other changes were due to the availability of a preferred regimen.

Virologic failure was common after ART-2 initiation, reaching a cumulative incidence of $25 \%$ at 5 years. Failure on ART-1 was a strong predictor of failure on ART-2: cumulative incidence of virologic failure 3 years after changing from ART-1 to ART-2 due to failure was nearly $45 \%$, and the hazard of virologic failure for these patients was twice that of those who changed to ART-2 due to toxicity. These results may in part reflect non-adherence. Regardless, patients failing an initial regimen are likely to fail a second regimen and should be monitored with extra care, perhaps through the re-allocation of clinic resources and staff to facilitate retention in care, medication adherence counseling, and more frequent laboratory monitoring, as mentioned above (45).

This study provides policy makers and funders with additional evidence that patients should be started on regimens with the greatest likelihood of enhancing CD4 recovery, maintaining viral suppression, and minimizing side-effects, thereby promoting efficient resource usage in resource-limited settings. This evidence is compelling because it includes data on reasons for regimen switches and granular details of the regimen constituents/classes associated with clinical outcomes among more than 5,500 patients in seven countries spanning more than 15 years. While addressing questions encountered by clinical care providers, this cohort study also contributes valuable information on the nature of HAART response among a particularly vulnerable population (those who require HAART regimen changes) in a lessstudied region that is still of major importance in the HIV/AIDS epidemic (46).

Our study has several weaknesses inherent to retrospective observational studies. Decisions to change ART were not controlled by investigators, classification of reasons for changing regimens were not uniform across sites, some data were missing and had to be imputed using multiple imputation techniques, many patients were lost to follow-up (rates of failure and death typically differ in those lost than those remaining in care [47-48]), and data on adherence or resistance genotyping were not collected. Differences in frequency of viral load monitoring across sites could lead to variability in identification of virologic failure. 
Our cohort includes urban sites and may not be representative of the respective countries or the region as a whole. Finally, the observation of higher risks of virologic failure after switching to regimens containing more potent drugs may be a symptom of confounding by indication, wherein patients doing poorly are those more likely to be placed on highly potent therapies (49). We attempted to reduce this confounding by adjusting all models using CD4 prior to ART-2 and CD4 change between ART-1 and ART-2.

In conclusion, in this large study of patients modifying their first antiretroviral regimen in Latin America and the Caribbean, significant risks of mortality, virologic failure, and further regimen change were observed. Many expanded access programs have focused on providing first-line regimens. Rates of modifications of these regimens most likely will remain high, whether due to virologic failure, concomitant medication interactions, or tolerability issues, suggesting more patients will require changes in first HAART, especially in resourcelimited settings where newer and less toxic drugs are still not the standard of care. The findings of our study provide evidence for such need and should be considered in the larger context of ART procurement policy.

\section{Supplementary Material}

Refer to Web version on PubMed Central for supplementary material.

\section{Acknowledgments}

This work was supported by the National Institute of Allergy and Infectious Diseases (NIAID) as part of the International Epidemiologic Databases to Evaluate AIDS (IeDEA): U01 AI069923.

We gratefully acknowledge all patients, caregivers, and data managers involved in the CCASAnet cohort.

\section{References}

1. Wada N, Jacobson LP, Cohen M, et al. Cause-specific life expectancies after 35 years of age for human immunodeficiency syndrome-infected and human immunodeficiency syndrome-negative individuals followed simultaneously in long-term cohort studies, 1984-2008. Am J Epidemiol. 2013; 177:116-25. [PubMed: 23287403]

2. Mills EJ, Bakanda C, Birungi J, et al. Life expectancy of persons receiving combination antiretroviral therapy in low-income countries: a cohort analysis from Uganda. Ann Intern Med. 2011; 155:209-16. [PubMed: 21768555]

3. van Sighem A, Gras LA, Reiss P, et al. ATHENA national observational cohort study. Life expectancy of recently diagnosed asymptomatic HIV-infected patients approaches that of uninfected individuals. AIDS. 2010; 24:1527-35. [PubMed: 20467289]

4. Antiretroviral Therapy Cohort Collaboration. Life expectancy of individuals on combination antiretroviral therapy in high-income countries: a collaborative analysis of 14 cohort studies. Lancet. 2008; 372:293-9. [PubMed: 18657708]

5. Johnson LF, Mossong J, Dorrington RE, et al. Life expectancies of South African adults starting antiretroviral treatment: collaborative analysis of cohort studies. PLoS Med. 2013; 10:e1001418. [PubMed: 23585736]

6. HIV Reporting. Global Update on the Health Sector Reponse to HIV. World Health Organization; 2014. Available at: http://www.who.int/hiv/pub/progressreports/update2014/en/ [Accessed 27 November, 2014]

7. UNAIDS. [Accessed 27 November, 2014] 15 million accessing treatment. Available at: http:// www.unaids.org/en/targetsandcommitments 
8. PAHO. Antiretroviral Treatment in the Spotlight:A Public Health Analysis in Latin America and the Caribbean, 2014. Washington, DC: PAHO; 2014.

9. The World Bank. [Accessed July 6, 2014] Country and Lending Groups. Available at: http:// data.worldbank.org/about/country-classifications

10. Grinsztejn B, Veloso VG, Friedman RK, et al. Early mortality and cause of deaths in patients using HAART in Brazil and the United States. AIDS. 2009; 23:2107-14. [PubMed: 19770698]

11. Tuboi SH, Schechter M, McGowan CC, et al. Mortality during the first year of potent antiretroviral therapy in HIV-1-infected patients in 7 sites throughout Latin America and the Caribbean. J Acquir Immune Defic Syndr. 2009; 51:615-23. [PubMed: 19430306]

12. Koenig SP, Rodriguez LA, Bartholomew C, et al. Long-term antiretroviral treatment outcomes in seven countries in the Caribbean. J Acquir Immune Defic Syndr. 2012; 59:60-71.

13. Braitstein P, Brinkhof MW, Dabis F, et al. Antiretroviral Therapy in Lower Income Countries (ART-LINC) Collaboration; ART Cohort Collaboration (ART-CC) groups. Mortality of HIV-1infected patients in the first year of antiretroviral therapy: comparison between low-income and high-income countries. Lancet. 2006; 367:817-24. [PubMed: 16530575]

14. Cesar C, Shepherd BE, Krolewiecki AJ, et al. Rates and reasons for early change of first HAART in HIV-1-infected patients in 7 sites throughout the Caribbean and Latin America. PLoS One. 2010; 5 (6):e10490. [PubMed: 20531956]

15. Cardoso SW, Grinsztejn B, Velasque L, et al. Incidence of modifying or discontinuing first HAART regimen and its determinants in a cohort of HIV- infected patients from Rio de Janeiro, Brazil. AIDS Res Hum Retroviruses. 2010; 26:865-74. [PubMed: 20672997]

16. Prosperi MC, Fabbiani M, Fanti I, et al. Predictors of first-line antiretroviral therapy discontinuation due to drug-related adverse events in HIV-infected patients: a retrospective cohort study. BMC Infect Dis. 2012; 12:296. [PubMed: 23145925]

17. Wright S, Boyd MA, Yunihastuti E, et al. Rates and factors associated with major modifications to first-line combination antiretroviral therapy: results from the Asia- Pacific region. PLoS One. 2013; 8(6):e64902. [PubMed: 23840312]

18. Hawkins C, Murphy RL. Management of antiretroviral failure and resistance in developing countries. Curr Opin HIV AIDS. 2009; 4:538-44. [PubMed: 20048723]

19. Abgrall S, Ingle SM, May MT, et al. Antiretroviral Therapy Cohort Collaboration (ART-CC). Durability of first ART regimen and risk factors for modification, interruption or death in HIVpositive patients starting ART in Europe and North America 2002-2009. AIDS. 2013; 27:803-13. [PubMed: 23719350]

20. Waters L, Bansi L, Asboe D, et al. Second-line protease inhibitor-based antiretroviral therapy after non-nucleoside reverse transcriptase inhibitor failure: the effect of a nucleoside backbone. Antivir Ther. 2013; 18:213-9. [PubMed: 23653911]

21. Bunupuradah T, Ananworanich J, Chetchotisakd P, et al. Etravirine and rilpivirine resistance in HIV-1 subtype CRF01_AE-infected adults failing non-nucleoside reverse transcriptase inhibitorbased regimens. AntivirTher. 2011; 16:1113-21.

22. Hill A, McBride A, Sawyer AW, et al. Resistance at virological failure using boosted protease inhibitors versus nonnucleoside reverse transcriptase inhibitors as first-line antiretroviral therapyimplications for sustained efficacy of ART in resource-limited settings. J Infect Dis. 2013; 207(suppl 2):S78-84. [PubMed: 23687293]

23. Etiebet MA, Shepherd J, Nowak RG, et al. Tenofovir-based regimens associated with less drug resistance in HIV-1-infected Nigerians failing first-line antiretroviral therapy. AIDS. 2013; 27:553-61. [PubMed: 23079810]

24. Murphy RA, Sunpath H, Lu Z, et al. Outcomes after virologic failure of first-line ART in South Africa. AIDS. 2010; 24:1007-12. [PubMed: 20397305]

25. Boyd MA, Kumarasamy N, Moore CL, et al. SECOND-LINE Study Group. Ritonavir-boosted lopinavir plus nucleoside or nucleotide reverse transcriptase inhibitors versus ritonavir-boosted lopinavir plus raltegravir for treatment of HIV-1 infection in adults with virological failure of a standard first-line ART regimen (SECOND-LINE): a randomised, open-label, non-inferiority study. Lancet. 2013; 381:2091-9. [PubMed: 23769235] 
26. Pujades-Rodríguez M, Balkan S, Arnould L, et al. Treatment failure and mortality factors in patients receiving second-line HIV therapy in resource-limited countries. JAMA. 2010; 304:30312. [PubMed: 20639564]

27. Ajose O, Mookerjee S, Mills EJ, et al. Treatment outcomes of patients on second- line antiretroviral therapy in resource-limited settings: a systematic review and meta-analysis. AIDS. 2012; 26:929-38. [PubMed: 22313953]

28. Abgrall, S.; Cornish, R.; Mugavero, M., et al. Outcomes after switch from or interruption to first ART regimen: The ART Cohort Collaboration. 18th Conference on Retroviruses and Opportunistic Infections; Boston. February 27-Mar 3, 2011;

29. McGowan CC, Cahn P, Gotuzzo E, et al. Cohort Profile: Caribbean, Central and South America Network for HIV research (CCASAnet) collaboration within the International Epidemiologic Databases to Evaluate AIDS (IeDEA) programme. Int J Epidemiol. 2007; 36:969-76. [PubMed: 17846055]

30. Schoni-Affolter F, Keiser O, Mwango A, et al. Estimating loss to follow-up in HIV-infected patients on antiretroviral therapy: the effect of the competing risk of death in Zambia and Switzerland. PLoS One. 2011; 6:e27919. [PubMed: 22205933]

31. Kalbfleisch, JD.; Prentice, RL. The Statistical Analysis of Failure Time Data. New York, NY: John Wiley \& Sons, Inc; 1980.

32. Little, RJA.; Rubin, DB. Statistical Analysis with Missing Data. 2. Wiley; 2002.

33. Howe CJ, Cole SR, Westreich DJ, et al. Splines for trend analysis and continuous confounder control. Epidemiology. 2011; 22:874-875. [PubMed: 21968779]

34. Grambsch PM, Therneau TM. Proportional hazards tests and diagnostics based on weighted residuals. Biometrika. 1994; 81:515-526.

35. Alave J, Paz J, González E, et al. Risk factors associated with virologic failure in HIV-infected patients receiving antiretroviral therapy at a public hospital in Peru. Rev Chilena Infectol. 2013; 30:42-8. [PubMed: 23450408]

36. Rajasekaran S, Jeyaseelan L, Vijila S, et al. Predictors of failure of first-line antiretroviral therapy in HIV-infected adults: Indian experience. AIDS. 2007; 21(suppl 4):S47-53. [PubMed: 17620752]

37. Paton, N.; Kityo, C.; Hoppe, A., et al. A pragmatic randomised controlled strategy trial of three second-line treatment options for use in public health rollout programme settings: the EuropeAfrica Research Network for Evaluation of Second-Line Therapy (EARNEST) Trial [WELBB02]. 7th International AIDS Society Conference on HIV Pathogenesis, Treatment, and Prevention (IAS 2013); Kuala Lumpur. June 30-July 3, 2013;

38. Hart E, Curtis H, Wilkins E, et al. National review of first treatment change after starting highly active antiretroviral therapy in antiretroviral-naïve patients. HIV Med. 2007; 8:186-191. [PubMed: 17461863]

39. Cicconi P, Cozzi-Lepri A, Castagna A, et al. Insights into reasons for discontinuation according to year of starting first regimen of highly active antiretroviral therapy in a cohort of antiretroviralnaïve patients. HIV Med. 2010; 11:104-13. [PubMed: 19732176]

40. [on 18 June, 2015] Guidelines for the use of antiretroviral agents in HIV-1-infected adults and adolescents. Downloaded from http://aidsinfo.nih.gov/guidelines

41. World Health Organization. [accessed June, 20, 2015] Consolidated guidelines on the use of antiretroviral drugs for treating and preventing HIV infection. Jun. $2013 \mathrm{http}: / /$ www.who.int/hiv/pub/guidelines/arv2013/download/en/index.html

42. Boettiger DC, Nguyen VK, Durier N, et al. Efficacy of second-line antiretroviral therapy among people living with HIV/AIDS in Asia: Results from the TREAT Asia HIV Observational Database. J Acquir Immune Defic Syndr. 2015; 68:186-95. [PubMed: 25590271]

43. Carrero-Gras A, Antela A, Muñoz-Rodríguez J, et al. Nuke-sparing regimens as a main simplification strategy and high level of toxicity resolution after antiretroviral switch: the SWITCHART Study. J Int AIDS Soc. 2014; 17(4 Suppl 3):19819. [PubMed: 25397563]

44. Amoroso A, Etienne-Mesubi M, Edozien A, et al. Treatment outcomes of recommended first-line antiretroviral regimens in resource-limited clinics. J Acquir Immune Defic Syndr. 2012; 60:31420. [PubMed: 22343178] 
45. Miller LG, Golin CE, Hays RD, et al. Impact of antiretroviral regimen switches on adherence. HIV Clin Trials. 2002; 3:355-60. [PubMed: 12407484]

46. Sabin CA. Cohort studies: to what extent can they inform treatment guidelines? Curr Opin Infect Dis. 2010; 23:15-20. [PubMed: 19996747]

47. Egger M, Spycher BD, Sidle J, et al. Correcting mortality for loss to follow-up: a nomogram applied to antiretroviral treatment programmes in sub-Saharan Africa. PLoS Med. 2011; 8(1):e1000390. [PubMed: 21267057]

48. Geng EH, Glidden DV, Bangsberg DR, et al. A causal framework for understanding the effect of losses to follow-up on epidemiologic analyses in clinic-based cohorts: the case of HIV-infected patients on antiretroviral therapy in Africa. Am J Epidemiol. 2012; 175:1080-7. [PubMed: 22306557]

49. Walker AM. Confounding by indication. Epidemiology. 1996; 7:335-6. [PubMed: 8793355] 

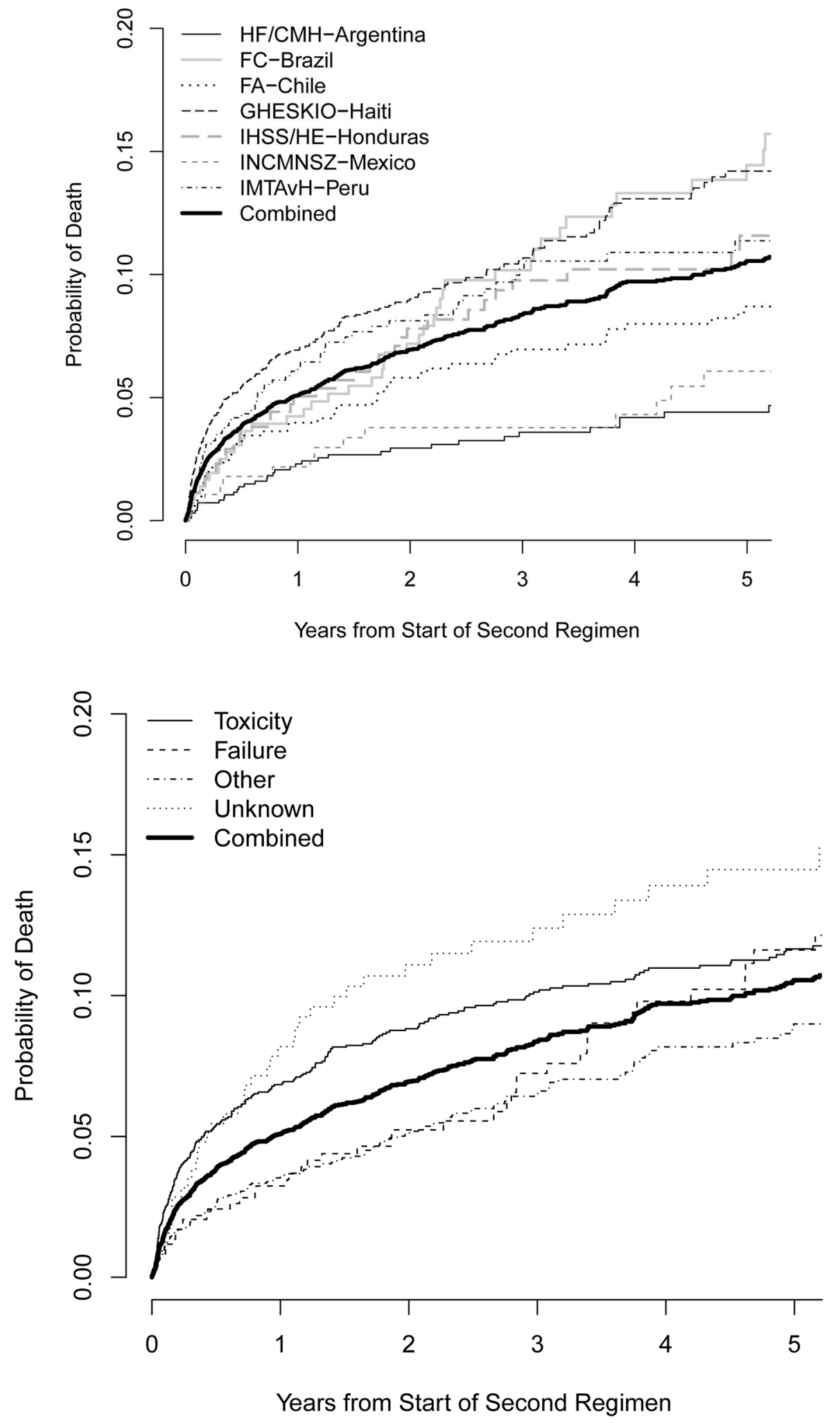

Figure 1. 
Estimated probability of death after start of second ART regimen (ART-2) by study site (a) and reason for starting ART-2 (b). 


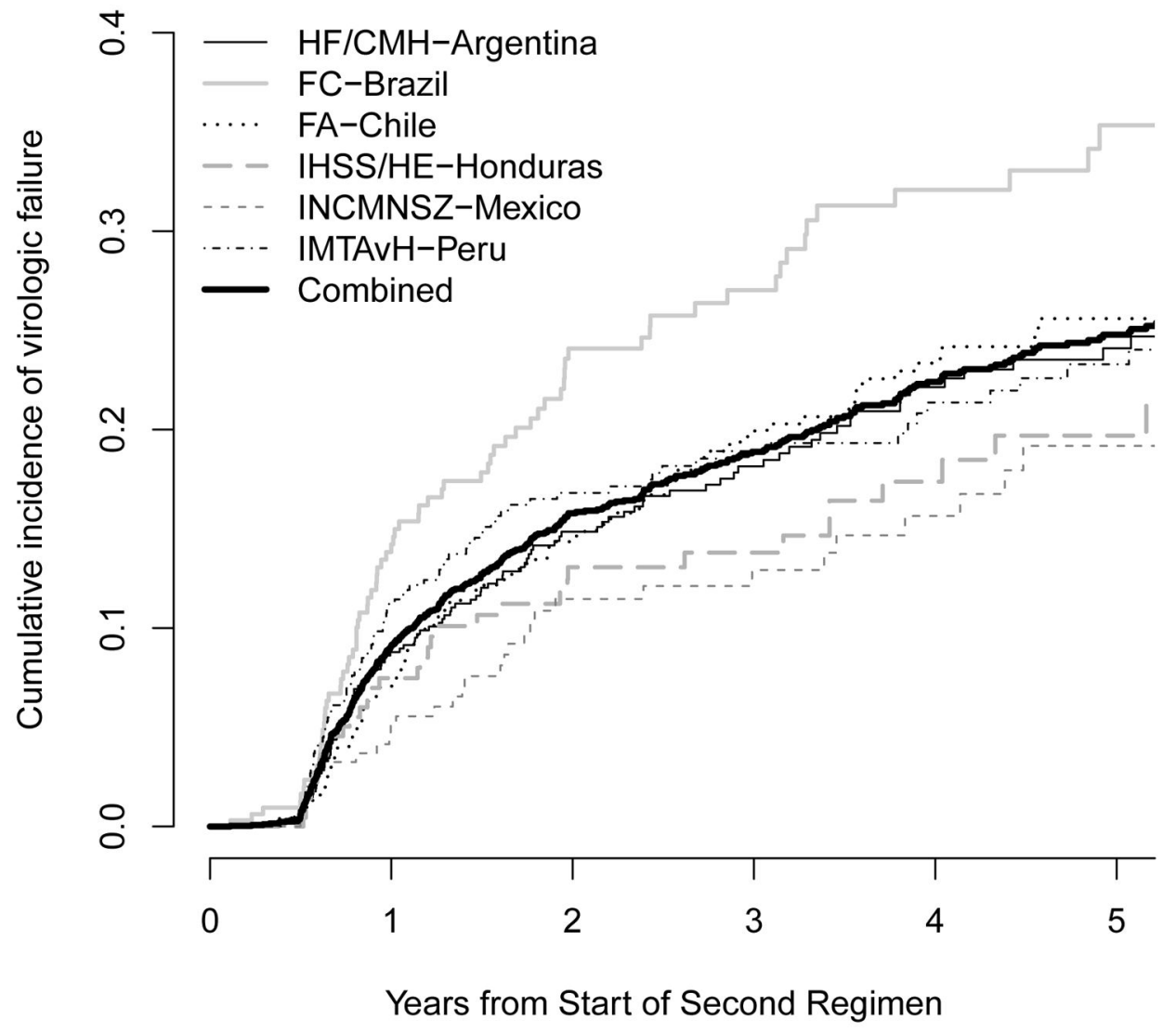




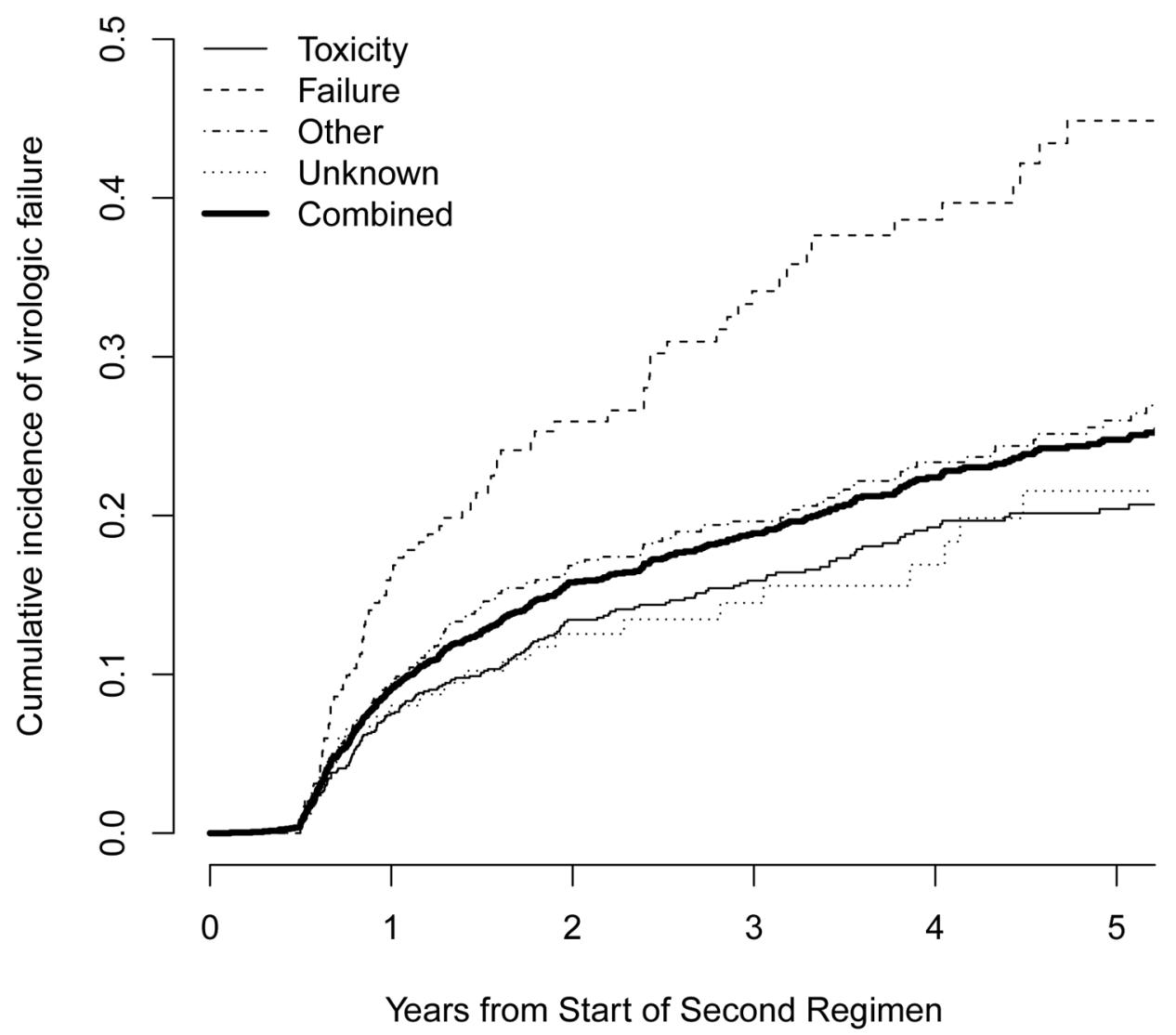

Figure 2.

Cumulative incidence of virologic failure after start of second ART regimen (ART-2) by study site (a) and reason for starting ART-2 (b). 


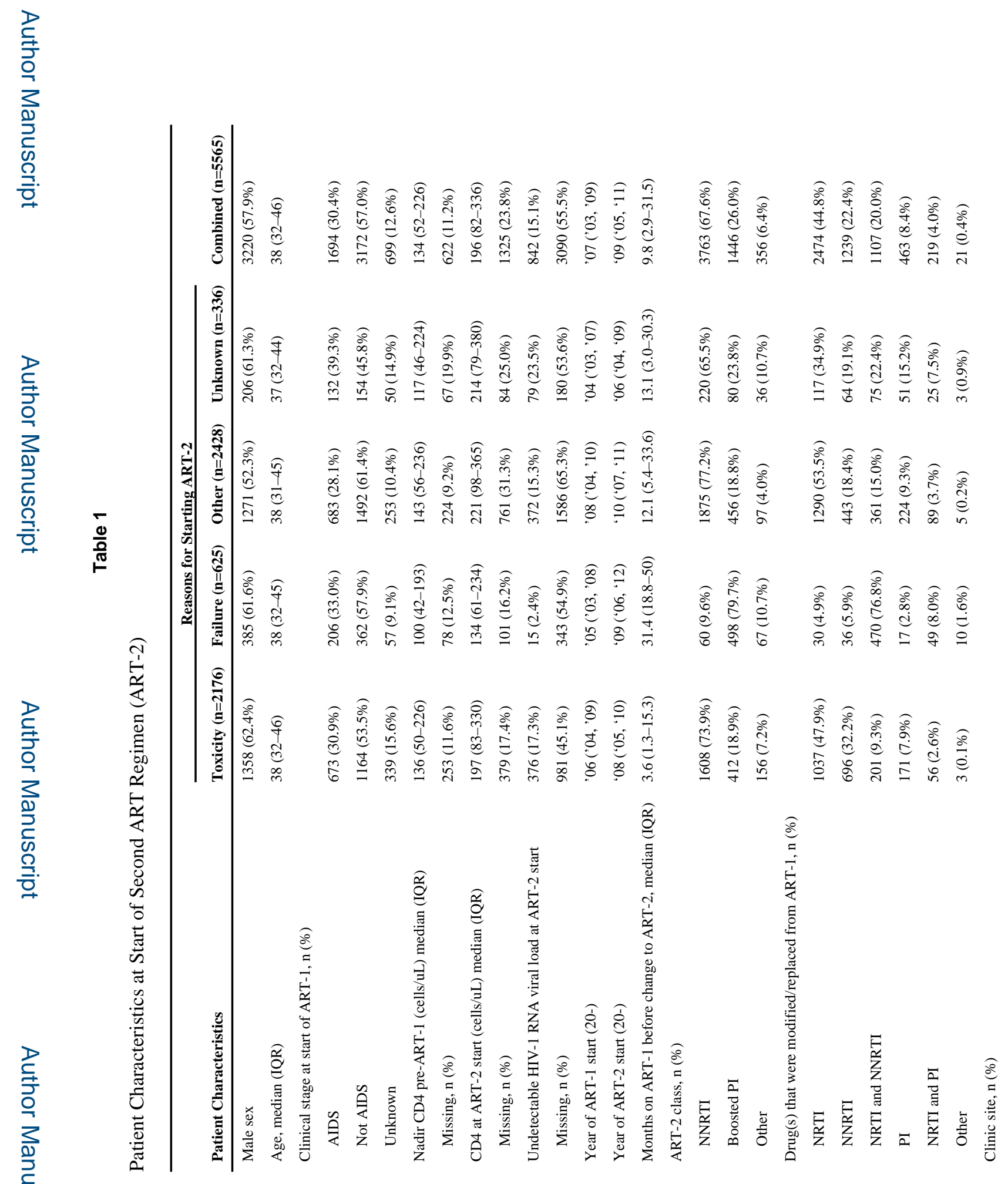

J Acquir Immune Defic Syndr. Author manuscript; available in PMC 2017 January 01. 


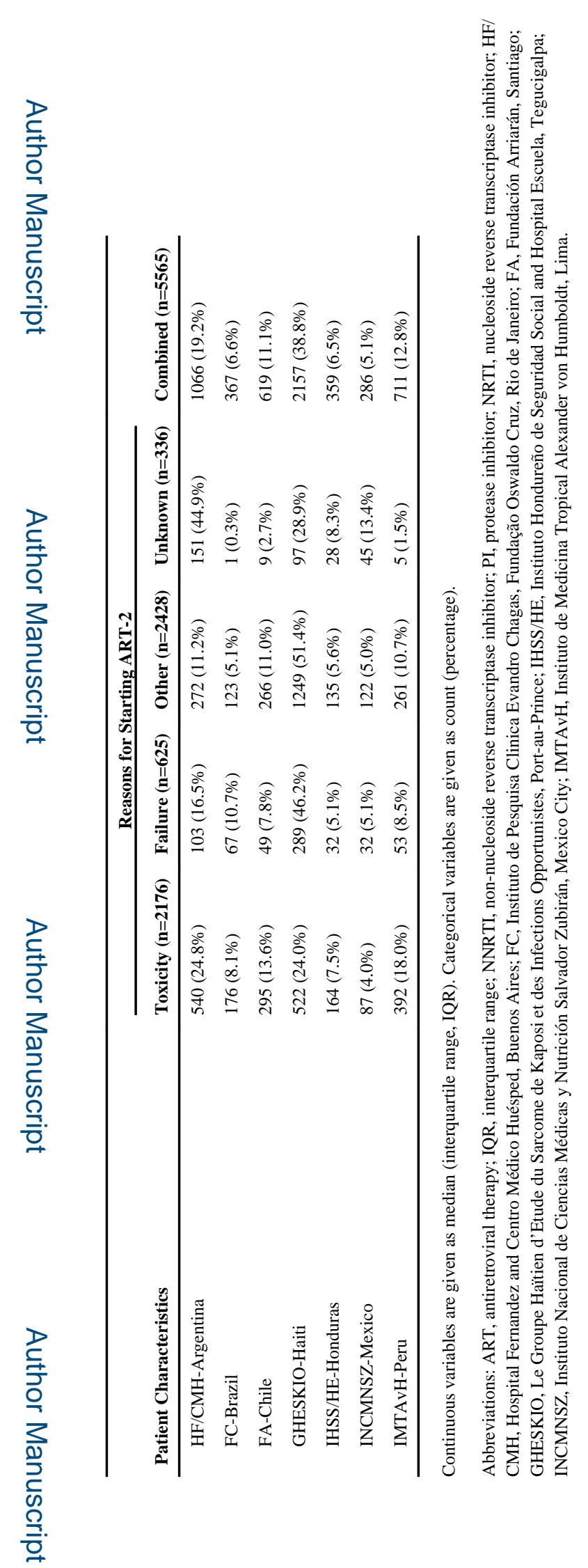

J Acquir Immune Defic Syndr. Author manuscript; available in PMC 2017 January 01. 
Table 2

Hazard Ratios for Death After Start of Second ART Regimen (ART-2). Of 5565 Patients Starting ART-2, 494 Died During a Median of 2.9 Years of Follow-up.*

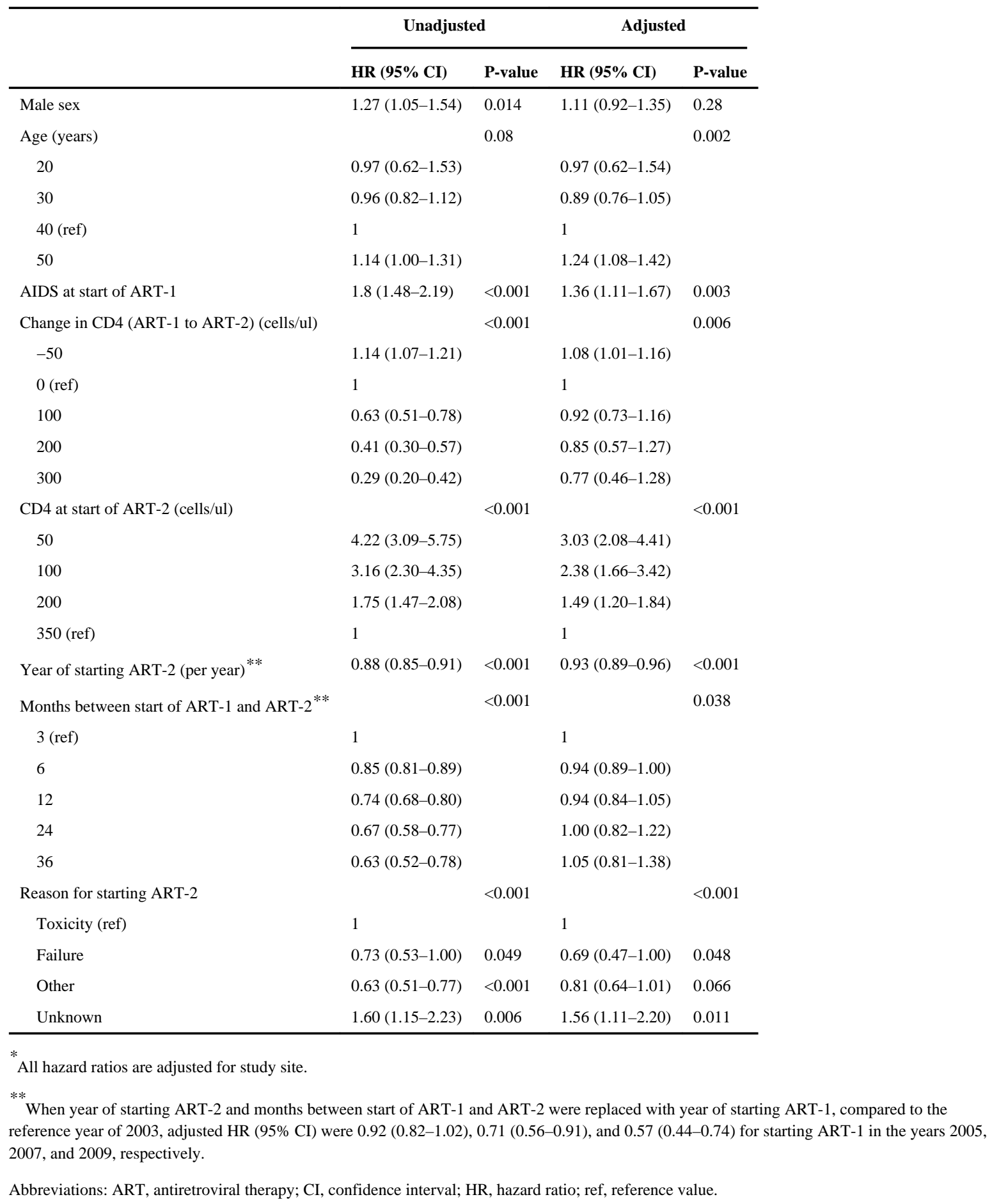

J Acquir Immune Defic Syndr. Author manuscript; available in PMC 2017 January 01. 
Table 3

Hazard Ratios for Virologic failure While on Second ART Regimen (ART-2). Of 5565 Patients Starting ART-2, 456 Experienced Virologic Failure During a Median of 2.9 Years of Follow-up.

\begin{tabular}{|c|c|c|c|c|}
\hline & \multicolumn{2}{|c|}{ Unadjusted } & \multicolumn{2}{|l|}{ Adjusted } \\
\hline & HR $(95 \% \mathrm{CI})$ & P-value & HR $(95 \%$ CI $)$ & P-value \\
\hline Male sex & $0.94(0.76-1.15)$ & 0.53 & $0.87(0.71-1.08)$ & 0.21 \\
\hline Age (years) & & $<0.001$ & & 0.001 \\
\hline 20 & $1.54(1.01-2.36)$ & & $1.51(0.98-2.32)$ & \\
\hline 30 & $1.13(0.95-1.35)$ & & $1.05(0.88-1.27)$ & \\
\hline 40 (ref) & 1 & & 1 & \\
\hline 50 & $0.75(0.64-0.89)$ & & $0.80(0.68-0.96)$ & \\
\hline AIDS at enrollment & $1.13(0.91-1.41)$ & 0.27 & $1.02(0.79-1.31)$ & 0.87 \\
\hline Change in CD4 (ART-1 to ART-2) (cells/ul) & & $<0.001$ & & $<0.001$ \\
\hline-50 & $1.16(1.09-1.24)$ & & $1.12(1.05-1.20)$ & \\
\hline 0 & 1 & & 1 & \\
\hline 100 & $0.72(0.62-0.84)$ & & $0.75(0.64-0.88)$ & \\
\hline 200 & $0.54(0.41-0.72)$ & & $0.57(0.40-0.82)$ & \\
\hline 300 & $0.43(0.31-0.59)$ & & $0.48(0.30-0.75)$ & \\
\hline CD4 at start of ART-2 (cells/uL) & & $<0.001$ & & 0.043 \\
\hline 50 & $2.21(1.66-2.93)$ & & $1.39(0.96-2.01)$ & \\
\hline 100 & $2.00(1.49-2.70)$ & & $1.28(0.90-1.83)$ & \\
\hline 200 & $1.50(1.23-1.83)$ & & $1.12(0.89-1.41)$ & \\
\hline 350 (ref) & 1 & & 1 & \\
\hline Year of starting ART-2 (per year) ${ }^{* *}$ & $0.93(0.90-0.96)$ & $<0.001$ & $0.95(0.92-0.99)$ & 0.008 \\
\hline Months between start of ART- 1 and ART-2** & & 0.024 & & 0.16 \\
\hline 3 (ref) & 1 & & 1 & \\
\hline 6 & $1.04(0.98-1.12)$ & & $1.06(0.98-1.14)$ & \\
\hline 12 & $1.02(0.92-1.13)$ & & $1.10(0.97-1.25)$ & \\
\hline 24 & $0.91(0.80-1.05)$ & & $1.12(0.93-1.36)$ & \\
\hline 36 & $0.83(0.70-1.00)$ & & $1.13(0.88-1.44)$ & \\
\hline Reason for starting ART-2 & & $<0.001$ & & $<0.001$ \\
\hline Toxicity (ref) & 1 & & 1 & \\
\hline Failure & $2.28(1.75-2.97)$ & $<0.001$ & $2.06(1.52-2.79)$ & $<0.001$ \\
\hline Other & $1.30(1.05-1.61)$ & 0.017 & $1.39(1.09-1.77)$ & 0.007 \\
\hline Unknown & $1.10(0.73-1.67)$ & 0.65 & $1.06(0.69-1.62)$ & 0.80 \\
\hline
\end{tabular}

J Acquir Immune Defic Syndr. Author manuscript; available in PMC 2017 January 01. 